Presented at the Orbit Correction and Analysis Workshop, BNL, Upton, New York, December 1-3, 1993.

\title{
Coupling Correction Using Closed Orbit Measurements *
}

\author{
J. Safranek and S. Krinsky \\ National Synchrotron Light Source \\ Brookhaven National Laboratory \\ Upton, NY 11973
}

February 18, 1994

\begin{abstract}
We describe a coupling correction scheme we have developed and used to successfully reduce the vertical emittance of the NSLS X-Ray ring by a factor of 6 to below $2 \dot{A}$. This gives a vertical to horizontal emittance ratio of less than $0.2 \%$. We find the strengths of 17 skew quadrupoles to simultaneously minimize the vertical dispersion and the coupling. As a measure of coupling we utilize the shift in vertical closed orbit resulting from a change in strength of a horizontal steering magnet. Experimental measurements confirm the reduced emittance.
\end{abstract}

\section{Introduction}

Betatron oscillations in the vertical plane of an electron storage ring can be excited in two ways - horizontal oscillations can be coupled into the vertical plane, or vertical oscillations can be excited directly when a photon is radiated where there is nonzero vertical dispersion, $\eta_{y}$. To reduce the vertical beam size, $\sigma_{y}$, one must correct both coupling and vertical dispersion. Meas rrements in the NSLS X-Ray ring indicate that $\eta_{y}$ and coupling give approximately equal contributions to $\sigma_{y}$.

When we started our work to reduce $\sigma_{y}$, the X-Ray ring had eight skew quadrupoles grouped in two families with a single power supply powering the four skews in each family. There was no method for correcting $\eta_{y}$. Now each of the eight original skew quadrupoles have individual power supplies, and nine new skew quads have been added. Four of the new skews are located in positions

"Work performed under the auspices of the U.S. Department of Energy

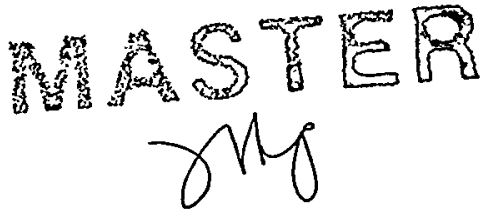


of high $\eta_{x}$, so they can be used to correct $\eta_{y}$ efficiently without exciting much coupling.

In the past the coupling on the X-Ray ring was minimized by adjusting the two families of skew quadrupoles to minimize the split between the transverse tunes at the difference resonance. With 17 individually powered skew quadrupoles, we needed a better method for measuring coupling than the tune split at the difference resonance. One such method has been developed at Cornell [1] in which the two transverse normal modes are excited on resonance, and the ellipse traced out by the betatron oscillations is measured at the beam position monitors. This method requires turn-by-turn beam position measurement capability which is not available with the present X-Ray ring hardware, so we developed another coupling correction algorithm that takes advantage of the high accuracy closed orbit monitors [2] in the X-Ray ring.

The approach we have adapted is to adjust the skew quadrupole strengths to minimize the vertical orbit distortion produced by changing the excitation of horizontal orbit correction dipole magnets, as well as to minimize the vertical dispersion. In this manner, we have reduced the vertical emittance down to 2 $\dot{A}$, corresponding to a vertical to horizontal emittance ratio of less than $0.2 \%$.

\section{Method of Reducing Vertical Beam Size}

Coupling: We sample the coupling by measuring the shifts in the vertical orbit, $\Delta y$, produced by varying the strengths, individually, of a set of horizontal steering magnets. Then we correct the coupling by determining the skew quadrupole strengths that minimize the vertical orbit shifts. We chose to simultaneously minimize 23 different vertical orbit shifts created by 23 different horizontal steering magnets distributed about the X-Ray ring. The vertical orbit distortion from a single steerer does not have all the coupling information. Skew quadrupoles located at positions of zero orbit distortion for that steering magnet do not create any vertical orbit distortion. Two horizontal steering magnets separated by close to an odd integer multiple of $90^{\circ}$ in horizontal betatron phase sample all the skew quadrupoles. We chose to use more than two steerers, because there is some vertical orbit shift that is simply due to imperfect rotational alignment of the steering magnets. This shift is not due to coupling and should not be corrected with the skew quadrupoles. We found that the most effective way to solve this problem is to look at the vertical orbit distortions from many horizontal steering magnets. The vertical distortions really caused by rotated quadrupoles are well corrected with the same skew quadrupole distribution for all the steerers, while the vertical orbit distortions from rotated steerers are randomly distributed and cannot be simultaneously corrected with the skews. We added horizontal steering magnets until we found that adding more no longer changed the skew quadrupole distribution derived to best correct the vertical orbit shifts. 


\section{DISCLAIMER}

Portions of this document may be illegible in electronic image products. Images are produced from the best available original document. 
Simulations with PATPET [3] confirmed that there is a strong correlation between the vertical orbit shift and the vertical emittance, and that reducing the vertical shift is an effective way to reduce the vertical emittance (figure 1). Both before and after the skew quadrupoles were adjusted to reduce the vertical orbit shifts, the calculated vertical emittance was found to exhibit the approximate dependence

$$
\epsilon_{y}(\dot{A}) \approx .002<\frac{\Delta y^{2}(\mu \mathrm{m})}{\beta_{y}(\mathrm{~m})}>
$$

where the shift in the vertical closed orbit, $\Delta y$, results from changing a horizontal steering magnet to give a $3 \mathrm{~mm}$ rms horizontal orbit distortion.

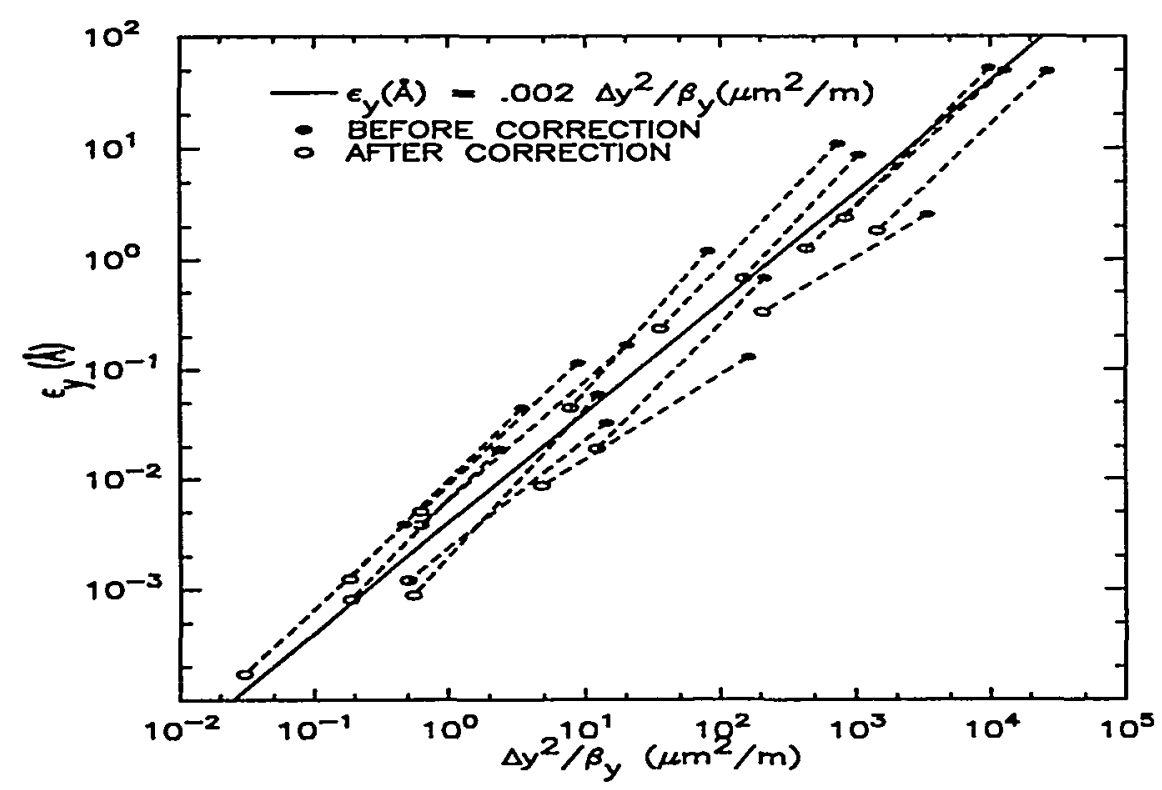

Figure 1: Simulation results from PATPET. Only coupling errors (no $\eta_{y}$ ) were introduced in the lattice. The dotted lines connect points for the same set of quadrupole rotation errors before and after correction.

VERTICAL DISPERSION: In order to minimize quantum excitation and thus minimize the vertical beam size, $\eta_{y}$ should be minimized in the bending magnets where the photons are radiated. In reference [4], the author shows that the emittance from dispersion is given by $\left.\epsilon_{y}=\frac{C_{g} \gamma^{2}}{\rho}<\mathcal{H}_{y}\right\rangle$, where $\left\langle\mathcal{H}_{y}\right\rangle$ is the average value of the Courant-Snyder invariant in the bending magnets. In reference [5] the author shows that $\left\langle\mathcal{H}_{y}\right\rangle$ is approximately equal to $\left.2<\frac{\eta_{y}^{2}}{\beta_{y}}\right\rangle$, so for the X-Ray ring ( $\rho=6.9 m$ and $\gamma=5057)$ the vertical emittance from 
dispersion is given by

$$
\epsilon_{y}(\dot{A}) \approx .03<\frac{\eta_{y}^{2}(\mathrm{~mm})}{\beta_{y}(\mathrm{~m})}>.
$$

Vertical dispersion can be excited by vertical bending or by skew quadrupoles at locations of nonzero $\eta_{x}$. We did not want to change the vertical closed orbit from the standard operational orbit in which the beamlines are steered, so we used skew quadrupoles at locations of large $\eta_{x}$ to correct the vertical dispersion.

CORRECTION ALGORITHM: The coupling and vertical dispersion correction was done simultaneously by solving a system of linear equations

$$
\mathrm{MK}=\mathrm{V}
$$

using singular value decomposition [6]. Here the vector $V$ has $48 \times 23+48$ elements. The first $48 \times 23$ elements are the measured vertical orbit shifts, $\Delta y / \sqrt{\beta}_{y}$, at the 48 beam position monitors for each of 23 horizontal steering magnets. The last 48 elements are the measured, $\eta_{y} / \sqrt{\beta}_{y}$, at the 48 BPMs. The $48 \times 23+48$ by 17 matrix $M$ is the measured change in $V$ with changes in the 17 skew quadrupoles. The linear equations are solved for $K$, the 17 skew strengths that minimize the rms of $\Delta y / \sqrt{\beta}_{y}$ and $\eta_{y} / \sqrt{\beta}_{y}$. The relative weight for correcting $\Delta y / \sqrt{\beta_{y}}$ versus $\eta_{y} / \sqrt{\beta}_{y}$ can be adjusted to give more or less correction of coupling versus vertical dispersion.

\section{Experimental Results}

The correction algorithm was very successful in reducing the vertical dispersion and vertical orbit shifts. We were able to reduce $\left\langle\frac{\Delta y^{2}}{\beta_{y}}>\right.$ by a factor of nine compared to the value $\left(5500 \mu \mathrm{m}^{2} / \mathrm{m}\right)$ achieved with the previous coupling correcton algorithm using only two families of skew quadrupoles. Figure 2 shows the reduction of $\Delta y / \sqrt{\beta}$ for a typical one of the sixteen horizontal orbit steering magnets. We simultaneously reduced $<\frac{\eta_{y}^{2}}{\beta_{y}}>$ by a factor of seven (figure 3 ) from a starting value of $300 \mathrm{~mm}^{2} / \mathrm{m}$.

According to equations 1 and 2 , we expect the reduction in $\left\langle\frac{\Delta y^{2}}{\beta_{y}}\right\rangle$ and in $\left\langle\frac{\eta_{y}^{2}}{\beta_{y}}\right\rangle$ to give a reduction in the vertical emittance of about eight. We measured the emittance reduction in three ways - 1) by measuring the decrease in Touschek lifetime [7] with approximately $80 \mathrm{~mA}$ stored in a single bunch, 2) by measuring the lifetime as a vertical scraper was moved into the beam, and 3 ) by directly measuring the vertical beam size reduction using $x$-ray pinhole cameras.

We saw nearly a factor of 2.5 decrease in the Touschek lifetime when we changed from the old skew quadrupole settings to the new ones. This indicates about a factor of 6 reduction in the vertical emittance. 


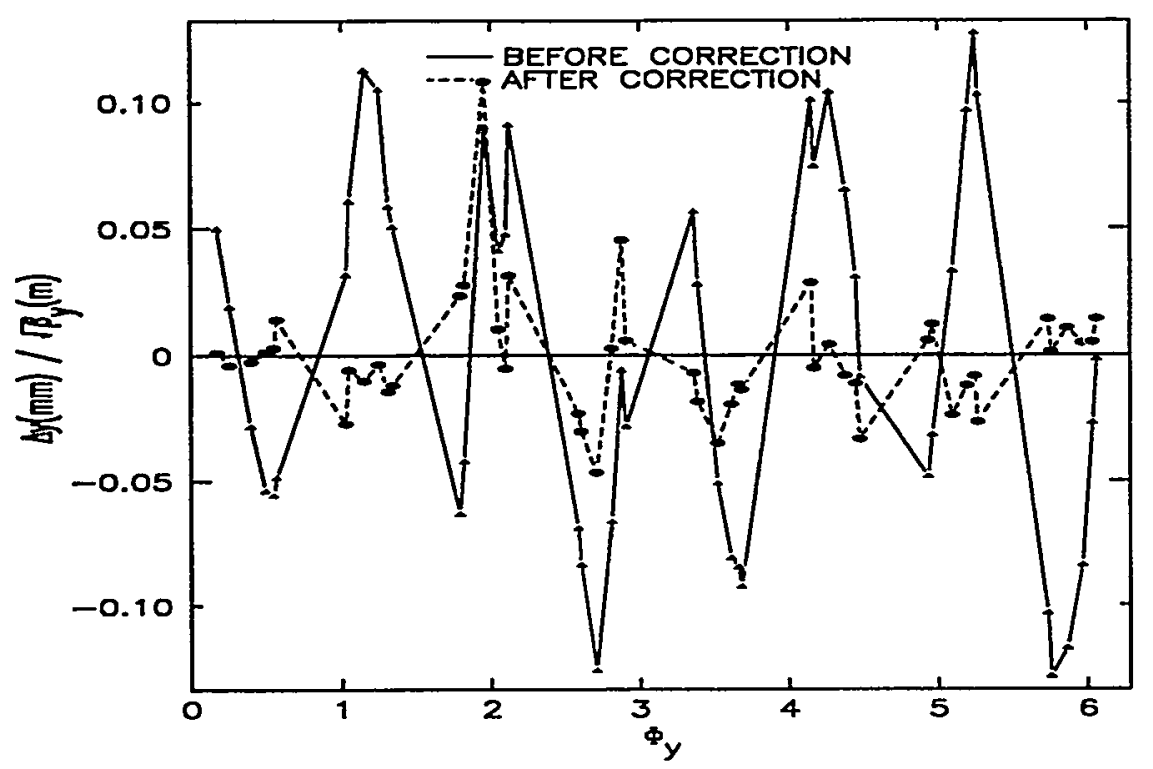

Figure 2: Measured vertical orbit shift resulting from a $3 \mathrm{~mm} \mathrm{rms}$ horizontal orbit shift from a single steerer.

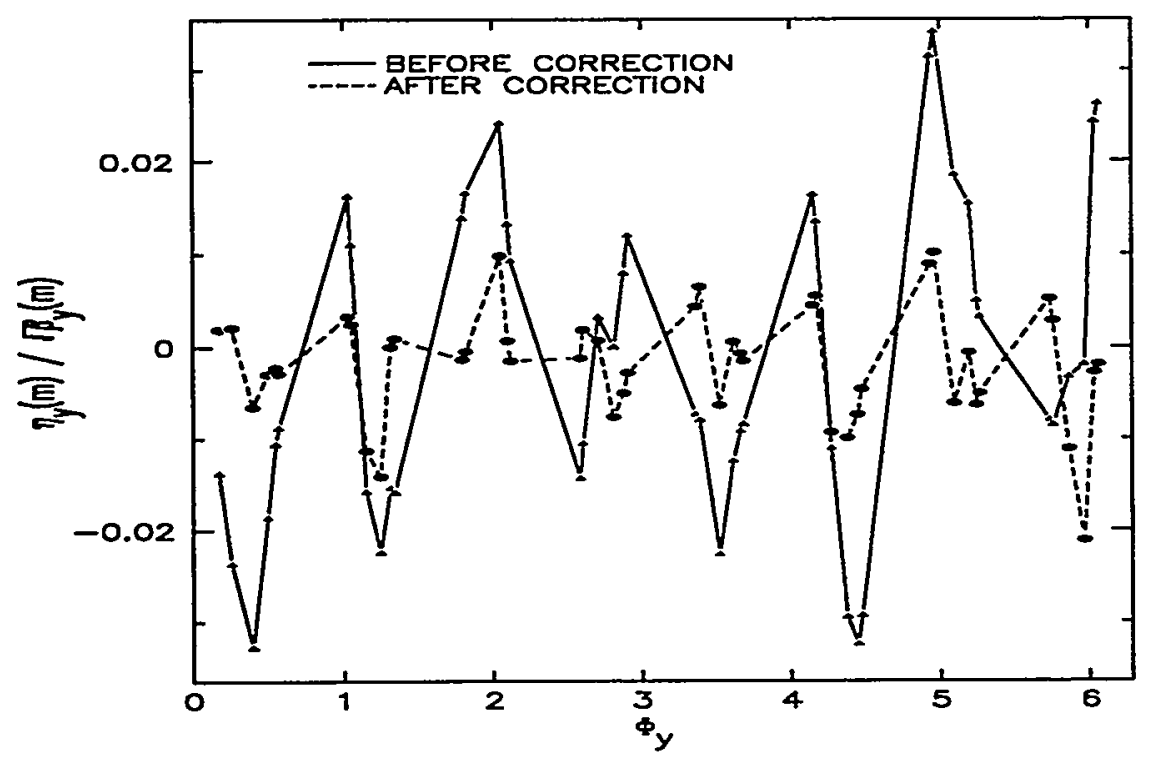

Figure 3: Measured vertical dispersion.

When a vertical scraper is close to the center of the stored beam, the quan- 
tum lifetime dominates the total lifetime of the stored beam. A fit to the lifetime vs. vertical scraper position for scraper positions close to the beam center gives a measure of the vertical beam size. Such a measurement indicated that the vertical beam size was approximately $20 \mu m$ with the old skew quadrupole settings at a location with $\beta_{y}=.4$. With the new skew quadrupole settings the measured beam size was approximately $7 \mu \mathrm{m}$ which implies a reduction in vertical emittance of about 8 .

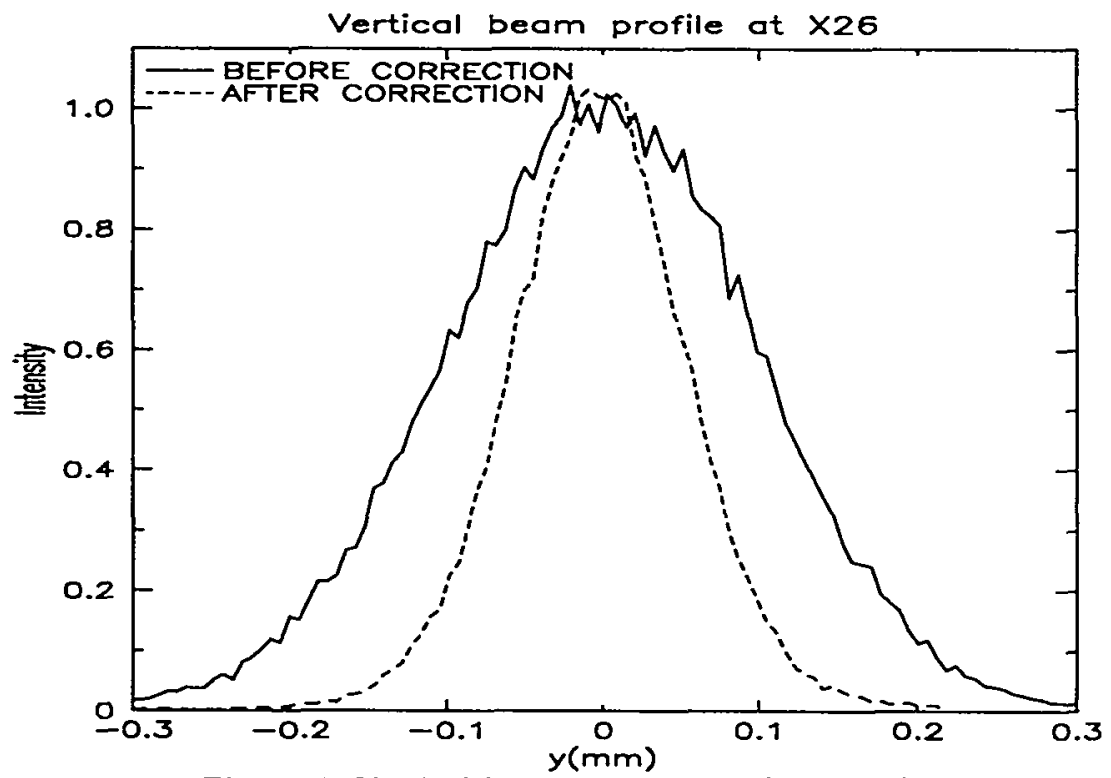

Figure 4: Vertical beam size at X26 $\left(\beta_{y}=15 \mathrm{~m}\right)$.

We measured the beam size reduction with $\mathrm{x}$-ray pinhole cameras on beamlines $\mathrm{X} 10$ and $\mathrm{X} 26$. The results with beamline X26 appeared to give the best resolution. Figure 4 shows the profile measured at X26 with the old skew quadrupole correction and the smaller profile with the new correction. Subtracting a small resolution factor in quadrature, these measurements indicate a reduction in electron beam size of 2 which gives a reduction in vertical emittance of 4.

The fact that a greater reduction in vertical beam size was indicated by the Touschek lifetime and scraper measurements than was measured with the x-ray pinhole camera may indicate that we are running into a resolution limit with the X26 pinhole camera. Work is continuing to better understand the resolution and absolute calibration of the pinhole cameras. 


\section{Acknowledgements}

We would like to express our sincere appreciation for the help we received measuring the beam size from M. Sansone, S. Sutton, and M. Rivers. Peter Stefan carried out the measurement and analysis of the lifetime vs. vertical scraper position. Discussions with R. Gluckstern were helpful when we were working to derive a correction algorithm.

\section{References}

[1] P. Bagley, D. Rubin, Proceedings 1989 IEEE Part. Accel. Conf. Chicago, IL, p. 874.

[2] R. Biscardi, J.W. Bittner, Proceedings 1989 IEEE Part. Accel. Conf. Chicago, IL, p. 15.

[3] L. Emery, H. Wiedemann, J. Safranek, SSRL/ACD note 36 revised, June, 1988.

[4] M. Sands, SLAC-121, November, 1970.

[5] T. Raubenheimer, SLAC-PUB-4937, February, 1990.

[6] W. Press, B. Flannery, S. Teukolsky, W. Vetterling, Numerical Recipes, Cambridge, 1990.

[7] H. Bruck, Circular Particle Accelerators, Los Alamos report LA-TR-72-10 rev. 